Document downloaded from:

http://hdl.handle.net/10251/51121

This paper must be cited as:

Satorre, M. A.; Leliwa-Kopystynski, J.; Santonja, C.; et ál. (2013). Refractive index and density of ammonia ice at different temperatures of deposition. Icarus. 225(1):703-708. doi:10.1016/j.icarus.2013.04.023.

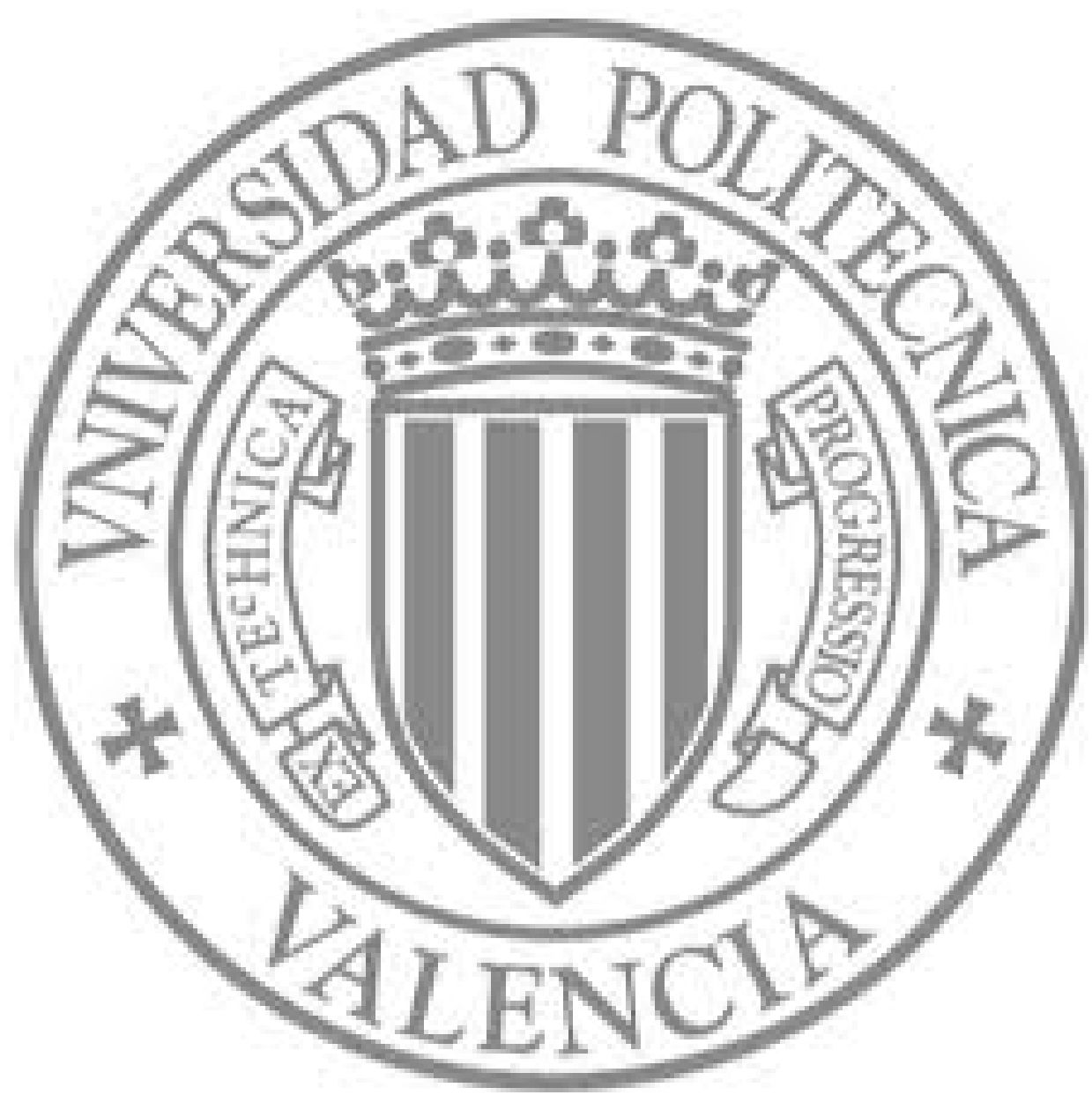

The final publication is available at

http://dx.doi.org/10.1016/j.icarus.2013.04.023

Copyright Elsevier 


\title{
Refractive index and density of ammonia ice at different temperatures of deposition
}

\author{
M. Á. Satorre*a, J. Leliwa-Kopystynski ${ }^{\mathrm{b}}$, C. Santonja ${ }^{\mathrm{a}}$, R. Luna ${ }^{\mathrm{a}}$ \\ ${ }^{a}$ Centro de tecnologías Físicas. Universitat Politècnica de València, 46022 Valencia, Spain \\ ${ }^{b}$ Warsaw University, Institute of Geophysics, Pasteura 7, 02-093 Warszawa, Poland
}

\begin{abstract}
Despite its relevance in astrophysical scenarios, optical properties and density of ammonia have been scarcely studied. This work presents new data on the real part of the refractive index of ammonia at $632.8 \mathrm{~nm}$ and density at different temperatures of deposition from $13 \mathrm{~K}$ up to its desorption temperature around $110 \mathrm{~K}$. The results show a significant variability for both parameters versus temperature, representing an increase of $50 \%$ for density and a $10 \%$ for refractive index as temperature increases in the range 13 - $60 \mathrm{~K}$, and at temperatures higher than $60 \mathrm{~K}$ a constant value is reached for both parameters. This initial variation and the further plateau reflects structural differences in its form at low and high temperatures that can play an important role in its interaction with other molecules of astrophysical interest.
\end{abstract}

Keywords:

Ices, Experimental techniques, Satellites surfaces, Trans-neptunian objects, Comets

\section{Introduction}

Ammonia ice is present in many astrophysical scenarios in Solar System bodies, on satellites as Enceladus (Emery et al., 2005; Verbiscer et al., 2006), Miranda (Bauer et al., 2002), on TNO's as Charon (Brown \& Calvin, 2000; Dumas et al., 2001; Cook et al., 2006, 2007), or Quaoar (Jewitt \& Luu, 2004), even in comets (Kawakita \& Watanabe (2002) and references therein). All these works confirm the prediction of Lewis (1972) who proposed ammonia as one of the most abundant molecules incorporated into the outer Solar System bodies, recently Pizzarello \& Williams (2012) confirmed the presence of ammonia from carbonaceous carbonites in the Early Solar System. Moreover ammonia modifies water properties, (Kargel, 1992; Lodders, 2003; Marion et al., 2012), even could be important not only for the surfaces but even for inner parts of icy satellites (Leliwa-Kopystynski et al., 2002). However, scarce studies have been performed on

\footnotetext{
*Corresponding author. Tel: +34966528542

Email address: msatorre@is.upv.es (M. Á. Satorre*)
}

the optical properties of this ice under astrophysical conditions (low pressure and low temperature). These studies are needed in a wide range of applications in planetary science and astrophysics to improve the understanding on the properties of these scenarios.

The theory of thin film optics has been described extensively in the literature (Born and Wolf, 1999; Heavens, 1991) and several techniques have been used to measure (in different spectral regions), the real part of the refractive index (herafter, $n$ ) (Tempelmeyer and Mills, 1968) .

Concerning ammonia, Romanescu et al.(2010) obtained a refractive index almost constant around 1.49 at three different temperatures of 80,90 and $100 \mathrm{~K}$. Other authors encountered some values at low temperatures $(20-30 \mathrm{~K})$ in the range 1.37-1.43 (Pipes et al., 1978; Wood \& Roux, 1982; Dawes et al., 2007), however, despite its relevance, no results for ammonia ice have been reported below $20 \mathrm{~K}$ and in the interval from $30 \mathrm{~K}$ up to $75 \mathrm{~K}$ (as far as we know). The main reason is probably its high reactivity that hampers its management in the laboratory.

Concerning density (hereafter $\rho$ ), experimental 
data are even more scarce, since the few reported 98 data are obtained at around $80 \mathrm{~K}$ or around 2599 $\mathrm{K}$. The results published so far show higher val- 100 ues when density is obtained at $80 \mathrm{~K}$. These results 101 obey the same behaviour than $n$ i.e.: significantly 102 lower values of density at low temperatures (20 K) ${ }_{103}$ (Wood \& Roux, 1982) respect that obtained around ${ }_{104}$ 80 K (Olovsson \& Templeton, 1959; Manzhelii \& Tolkachev, 1964; Wood \& Roux, 1982).

Differences in both, $n$ and $\rho$, at low and high 106 temperatures invite to infer different structures. ${ }_{107}$ During decades authors agree about an amorphous 108 structure at low temperatures and a crystalline one 109 at high temperatures. But no agreement is achieved 110 about a likely metastable structure that seems to 111 appear in a different range of temperatures depend- 112 ing on the authors. To clarify this controversial question, two different experimental works (Zheng \& Kaiser, 2007; Dawes et al., 2007) has been re- ${ }^{114}$ cently performed and both conclude in their results 115 the existence of only two different structures: an ${ }^{116}$ amorphous one below around $60 \mathrm{~K}$ (hereafter low ${ }^{117}$ temperatures means below $60 \mathrm{~K}$ ) and a crystalline ${ }^{118}$ one for temperatures higher than $60 \mathrm{~K}$ (hereafter 119 high temperatures means above $60 \mathrm{~K}$ ).

To help to disentangle the problem of the differ- ${ }^{121}$ ent structures of ammonia (amorphous, metastable ${ }^{122}$ and crystalline phase), it would be relevant finding ${ }^{123}$ additional values of $n$ and $\rho$ for different tempera- 124 tures of those present in the literature. $\quad 125$

On the other hand $n$ and $\rho$ are relevant by them- 126 selves for different. If the refractive index of a ${ }^{127}$ species is known, it is possible to calculate the thick- ${ }^{128}$ ness of a deposited film from the number of interfer- ${ }^{129}$ ence fringes obtained with a coherent light beam. In 130 our experiments we determine $n$ at $632.8 \mathrm{~nm}$ (He- ${ }^{131}$ Ne laser). This data is especially useful because the ${ }^{132}$ He-Ne wavelength is commonly used as a standard ${ }^{133}$ in almost any laboratory. Thickness is needed in ${ }^{134}$ the determination of another very important phys- 135 ical characteristic as density, which is relevant in 136 the assessment of many magnitudes of astrophysi- ${ }^{137}$ cal interest as the integrated absorbance strength, 138 porosity, penetration depth of ions impinging an 139 ice, etc.

In the case of experiments of irradiation, the pen- ${ }^{141}$ etration depths depends directly on density and ${ }^{142}$ determine whether an experiment is a thin (ions ${ }^{143}$ passing the film) or thick (ions stopped in the film) ${ }^{144}$ film experiment, by determining the stopping power ${ }^{145}$ with specialized programs as Ziegler's SRIM pro- ${ }^{146}$ gram (Ziegler et al. (1985)).
Density is also important in the analysis of many results: observational, experimental and theoretical. The integrated absorbance value $A(\mathrm{~cm}$ molecule ${ }^{-1}$ ), can be used to estimate column densities of ice constituents from the absorbtion spectra of the ices (Hudgins et al., 1993). Integrated absorbance is defined as:

$$
A=\frac{1}{C l} \int_{\nu(1)}^{\nu(2)} \tau_{\nu} d \nu
$$

where $C$ is the concentration of the absorbers, often expressed as molecule $\mathrm{cm}^{-3}$ (or mol $\mathrm{l}^{-1}$ ), $l$ is the path-length of the beam through the material, $\nu$ is the frequency in $\mathrm{cm}^{-1}, \tau_{\nu}=\ln (I o / I)$ is the ratio of the incident to the transmitted beam, with the integration performed over the band. $C$ is obtained as follows:

$$
C=\frac{\rho N_{A}}{M r}
$$

where $\rho$ is the density of the ice in $\mathrm{g} \mathrm{cm}^{-3}, N_{A}$ is the Avogadro constant in molecule $\mathrm{mol}^{-1}$ and $M r$ is the molecular mass in $\mathrm{g} \mathrm{mol}^{-1}$. d'Hendecourt \& Allamandola (1986) assumed that, for the calculation of $A$, the largest uncertainties arises from evaluating $\mathrm{C}$ (concentration of absorbers), as the densities of ices are not accurately known. They assumed the densities to be constant $\left(1 \mathrm{~g} \mathrm{~cm}^{-3}\right)$. This assumption was also made by many other authors, among them is used by Hudgins et al. (1993) in their exhaustive work to determine optical constants and integrated absorbance for mid- and farinfrared spectroscopy of ices.

Density is even relevant by itself in the physical chemistry of ices. The course of chemical differentiation depends very much on the relative densities of important phases and whether they tend to sink or float (Kargel, 1991). This author presents densities of representative cryovolcanic liquids and solids versus temperature. Knowing the density at temperatures relevant for astrophysical scenarios helps to understand part of their surface dynamics.

We present in this work new results on the real part of refractive index at $632.8 \mathrm{~nm}$ and bulk density of $\mathrm{NH}_{3}$ at different temperatures (ranging from 10 to $100 \mathrm{~K})$. These results can be used to better reproduce irradiation, integrated absorbances and buoyancy of ices at different temperatures and could help to better understand the structure of ammonia. In this article, a review of the experimental setup and procedures is described in the next section. In section 3 the experimental results are showed and discussed, and finally in section 4 the conclusions are exposed. 


\section{Experimental}

The experimental apparatus and the procedure 179 to obtain the refractive index and density is briefly 180 summarized here. A more detailed explanation in 181 some aspects is presented in Satorre et al. (2008). ${ }^{182}$ A simple schematic of the apparatus is shown in 183 Figure 1.

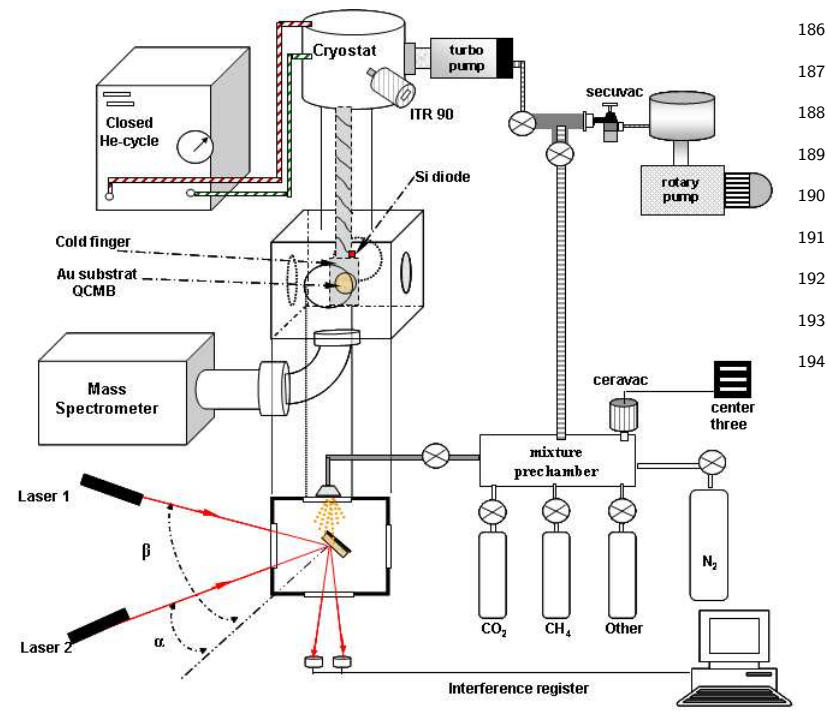

Figure 1: Experimental setup

We have measured refractive index and density of $\mathrm{NH}_{3}$ ice under high vacuum conditions (base pressure $<10^{-7}$ mbar) and temperature ranging from 13 to $100 \mathrm{~K}$.

Sample holder (quartz crystal microbalance, hereafter $\mathrm{QCMB}$ ) temperature is operated by the Intelligent Temperature Controller ITC 503S (Oxford Instruments). It uses the feedback of a silicon diode sensor (Scientific Instruments) located just beside the quartz, that allows the temperature to vary between 10.0 and $300.0 \pm 0.5 \mathrm{~K}$, by means a resistive heater.

Refractive index has been obtained by double laser interferometry at $632.8 \mathrm{~nm}$. This technique has been widely used in the literature as for example in the work of Tempelmeyer and Mills (1968).

The QCMB is used to calculate the mass of ice accreted per unit area (in $\mathrm{g} \mathrm{cm}^{-2}$ ). This value is obtained from the QCMB variation in frequency by using the Sauerbrey equation: $\Delta f=-S \cdot \Delta m$. In this equation $\Delta f$ is variation in frequency, $\Delta m$ represents the mass accreted onto the balance and $S$ is a specific constant for every QCMB. From the value obtained with the QCMB signal and the thickness, density is obtained.

During deposition, gas flows from the prechamber to the deposit vacuum chamber controlled by a needle valve (Leybold D50968). To form the ice film, a constant rate of deposition is used (around 1 $\mu \mathrm{m} \mathrm{h}^{-1}$ ), maintaining almost constant the aperture of the valve. $\mathrm{NH}_{3}$ enters the deposition chamber and deposit on the coldest parts growing a film onto the QCMB. The pressure of ammonia and contaminants composition during film growth is checked through the Quadrupole mass spectrometer (QMS) (AccuQuad RGA 100 with a resolution of $\sim 0.5$ amu). Some experiments, at different temperatures, are repeated to check its reproducibility and all the results are within the error bars. Ammonia used is Praxair 99.999.

Table 1: Density $(\rho)$ and refractive index $(n)$, obtained for $\mathrm{NH}_{3}$ at different temperatures of deposition compared with the literature.

\begin{tabular}{|c|c|c|c|c|}
\hline \\
\hline \multirow[t]{2}{*}{ T. (K) } & \multicolumn{2}{|c|}{$\begin{array}{c}\text { Density } \\
\left(\mathrm{g} \mathrm{cm}^{-3}\right)\end{array}$} & \multicolumn{2}{|c|}{$\begin{array}{l}\text { Refractive } \\
\text { index }\end{array}$} \\
\hline & $\rho \pm 5 \%$ & Lit. & $\mathrm{n} \pm 2.5 \%$ & Lit. \\
\hline 13 & 0.67 & & 1.38 & \\
\hline 20 & 0.72 & $0.76^{a}$ & 1.40 & $\begin{array}{l}1.37^{a} \\
1.42^{b}\end{array}$ \\
\hline 25 & & & & $1.44^{c}$ \\
\hline 40 & 0.80 & & 1.45 & \\
\hline 60 & 0.89 & & 1.49 & \\
\hline 75 & & & & $1.48^{c}$ \\
\hline 77 & & $0.861^{d}$ & & \\
\hline 80 & 0.87 & $0.87^{a}$ & 1.49 & $\begin{array}{l}1.41^{a} \\
1.49^{e} \\
1.42^{b}\end{array}$ \\
\hline 81 & & $0.863^{f}$ & & \\
\hline 90 & 0.90 & & 1.48 & $1.50^{e}$ \\
\hline 100 & 0.85 & & 1.48 & $\begin{array}{l}1.48^{e} \\
1.4^{c}\end{array}$ \\
\hline \multicolumn{5}{|c|}{$\begin{array}{l}\text { References } \\
\text { a: Wood and } \operatorname{Roux}(1982) \\
\text { b: Pipes et al.(1978) } \\
\text { c: Dawes et al. }(2007) \\
\text { d: Olovsson and Templeton(1959) } \\
\text { e: Romanescu et al.(2010) } \\
\text { f: Manzhelii and Tolkachev }(1964)\end{array}$} \\
\hline
\end{tabular}




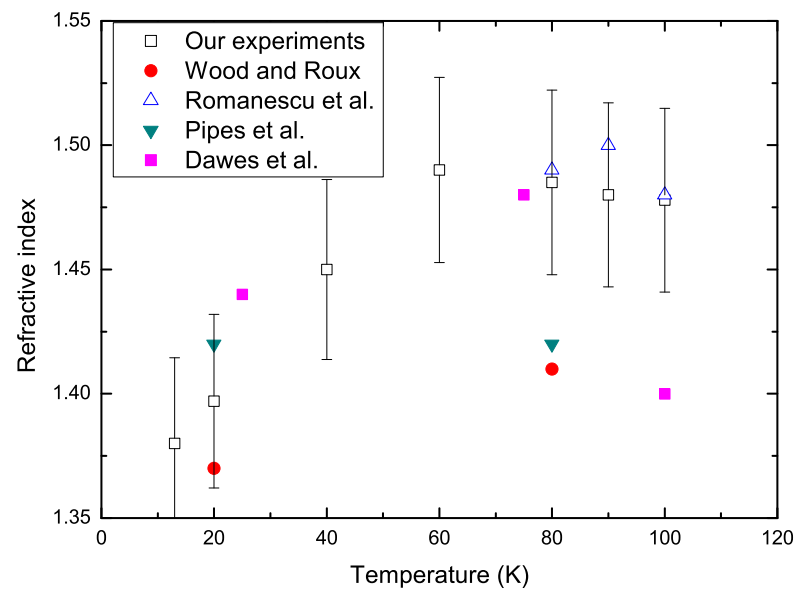

Figure 2: $\mathrm{NH}_{3}$ refractive index at different temperatures of deposition compared with data obtained from the literature.

\section{Results and discussion}

The motivation of our work is to provide new data for $n$ and $\rho$ at temperatures at which they are ${ }^{229}$ no previously obtained. These data are useful be- ${ }^{230}$ cause from previous results is clear that ammonia ${ }^{231}$ ice structure depends on the temperature of depo- ${ }^{232}$ sition. In this section we discuss the validity of our ${ }^{233}$ results comparing with experimental data obtained ${ }^{234}$ with both, the same and other experimental tech- ${ }^{235}$ niques.

Density and refractive index obtained at different ${ }^{237}$ temperatures in our set of experiments (ranging $13{ }^{238}$ to $100 \mathrm{~K}$ ), has been collected and presented in Table ${ }^{239}$ 1 together with other values taken from the liter- 240 ature, in order to compare them. Additionally, all ${ }^{241}$ the data for $n$ and $\rho$ have been plotted in Figure $2{ }^{242}$ and 3 respectively. The errors considered in these ${ }^{243}$ graphs are $2.5 \%$ for $n$ and $5 \%$ for $\rho$. Errors in the ${ }^{244}$ temperature are not marked since they are within ${ }_{245}$ the symbol size.

Our experimental data show two different be- ${ }^{247}$ haviours, below and above $60 \mathrm{~K}$. Below it (left side ${ }_{248}$ in Figures 2 and 3), $n$ and $\rho$ vary with temperature 249 as $n=(1.35+0.00231 \mathrm{~T})$ and $\rho=(0.628+0.00462250$ T) $\mathrm{g} \mathrm{cm}^{-3}$, with $n$ growing from 1.38 to 1.48 and $\rho{ }_{251}$ increasing from 0.67 to 0.87 . In both cases a plateau ${ }_{252}$ is reached for temperatures higher than $60 \mathrm{~K}$ (right ${ }_{253}$ side in Figures 2 and 3$)$, being $n_{\text {plateau }}=1.48 \pm{ }_{254}$ 0.05 , and $\rho_{\text {plateau }}=0.67 \pm 0.02 \mathrm{~g} \mathrm{~cm}^{-3}$. If we 255 substitute, in the straight fits, the plateau values of 256 $n$ and $\rho$ versus temperature is reached, we obtain ${ }^{257}$ a temperature for transition of $58 \pm 5 \mathrm{~K}$. These ${ }^{258}$

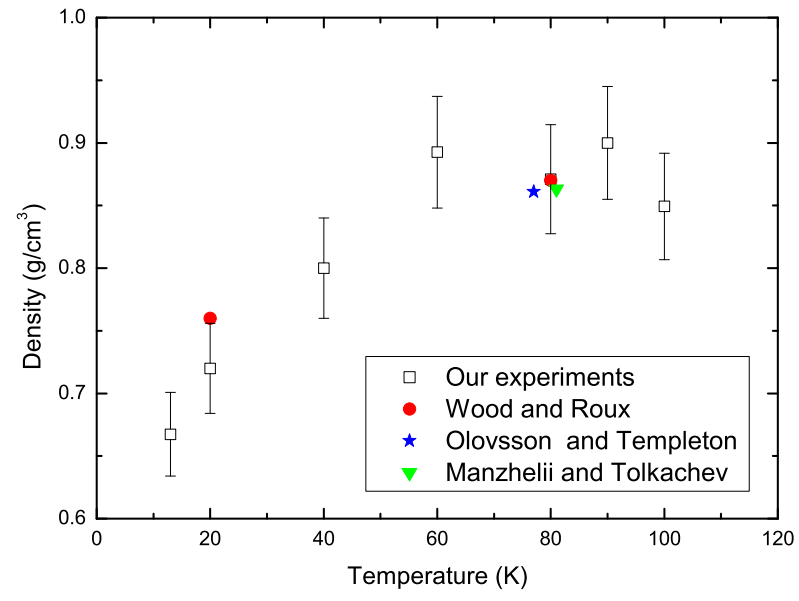

Figure 3: $\mathrm{NH}_{3}$ density at different temperatures of deposition compared with data obtained from the literature.

order for ammonia molecules below $60 \mathrm{~K}$ and a stable structure above it.

These results complete those published so far and agree with most of them (Figures 2 and 3). Only deviations around $5 \%$ are observed for $n$ at temperatures around $75 \mathrm{~K}$ (Figure 2). This particular discrepancy will be discussed in more detail later.

Looking at the literature data, the mail conclusion is that around $25 \mathrm{~K}$, values obtained for both $n$ and $\rho$ are lower than those obtained at around 80 $\mathrm{K}$ or higher. Because the lack of data below $25 \mathrm{~K}$ and from 30 up to $80 \mathrm{~K}$ it is no possible to establish a relationship between $n$ and $\rho$ and the structure of ammonia. Only three constant $n$ values presented by Romanescu et al. (2010) at 70, 80 and $90 \mathrm{~K}$ let us deduce a stable structure.

Refractive index values are usually obtained by double laser interferometry. Dawes et al. (2007) used a technique explained in detail by Westley et al. (1998) based on the interference curves with one laser. Among all these results (including ours), those obtained by Romanescu et al. (2010) by double laser interferometry are probably the most accurate due to their number of experiments for a specific temperature (16 experiments, at $50 \mathrm{~K}$ ) and because they analyze and subtract the output of the laser signal (using an additional sensor) correcting the artifacts of the device. This procedure presents the advantage that correcting the variation in the laser beam, the interference curves are clarified. Our results, taking into account the error bars, 
fully agree with the results of Dawes et al. (2007) at ${ }_{311}$ $77 \mathrm{~K}$, and that of Romanescu et al. (2010) at 80, 90312 and $100 \mathrm{~K}$ (see Figure 2). Dawes et al. (2007) work 313 under UHV conditions and their results at $100 \mathrm{~K} 314$ could be affected because under UHV conditions, 315 depending on the pressure of deposition, two pro- 316 cesses could compete in the dynamics of the pro- 317 cess, deposition and sublimation.

Pipes et al. (1978) and Wood \& Roux (1982) use 319 both almost the same experimental setup. As it 320 is clearly shown in Figure 2, at high temperatures 321 both works obtain lower values than any other au- 322 thors (including the result obtained in this work) 323 but at $20 \mathrm{~K}$ their results are within the error bars of ours. The discrepancy between the results obtained by these authors at high temperatures and all the others, is explained by Romanescu et al. (2010) from the deposition rate in the experiments of Pipes et al. (1978) and Wood \& Roux (1982) (in the order of $\left.\mu \mathrm{m} \mathrm{min}{ }^{-1}\right)$.

The explanation of the discrepancy, more evident for crystalline than for amorphous structure, could be that forming any crystalline structure, in the case of ammonia a Face Centered Cubic structure (thereafter FCC, see Figure 4) needs a certain experimental conditions. At higher temperatures than $60 \mathrm{~K}$ a crystalline structure is formed if deposition conditions allow it. A new molecule arriving needs a certain time to achieve the appropriate position and orientation. For almost all the experiments performed by many authors with different techniques and different rate of deposition, all of them conclude that no dependence of the rate of deposition is observed, but it seems that this is true up to a certain threshold. If it is exceeded, molecules have no time to reorganize in a crystalline structure. This threshold should be around that rate used by Pipes et al. (1978) and Wood \& Roux (1982) as Romanescu et al. (2010) point out as the cause of 325 the discrepancy between their results and those of 326 Pipes et al. (1978), Wood \& Roux (1982). If deposi- 327 tion rate finally influences on the result, this effect 328 should be more relevant for the crystalline phase ${ }_{329}$ than for amorphous ice, this would explain why at 330 $25 \mathrm{~K}$ their data agree with the other ones. 331

Concerning $\rho$ values, all the results for all the ${ }_{332}$ temperatures present in the literature fully agree 333 with our results (see Figure 3), that of Wood \& 334 Roux (1982), at 20 and $80 \mathrm{~K}$, using the same ex- 335 perimental technique of us, Manzhelii \& Tolkachev 336 (1964) using picnometer, and Olovsson \& Temple- ${ }_{337}$ ton (1959) using X-ray diffraction at around $80 \mathrm{~K} .{ }_{338}$
Romanescu et al. (2010) claim the high accuracy of the experimental technique using picnometers and X-ray diffraction for the $\rho$ values. Our results are within $1 \%$ to those last works, and, despite the difficulties of working with the QCMB (mentioned by Romanescu et al. (2010)).

Our results provide for the first time, enough additional data to show a clear linear increase in $n$ and $\rho$ values from low temperatures up to $60 \mathrm{~K}$ and additionally they confirm the presence of a steady state above $60 \mathrm{~K}$. Then all the experimental results point to, at least, two different structures, below and above $60 \mathrm{~K}$.

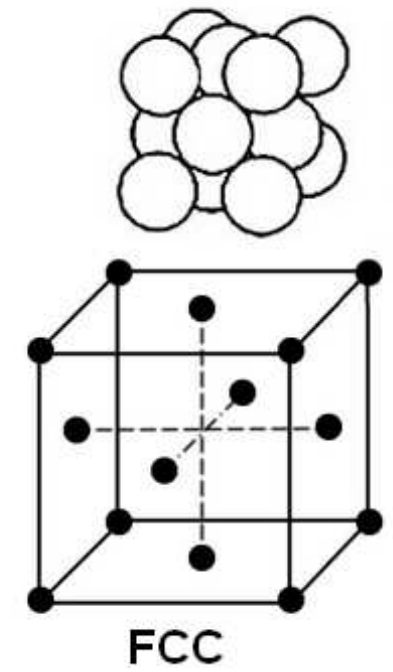

Figure 4: Crystal structure for $\mathrm{NH}_{3}$.

\section{Conclusions}

In this work the density and refractive index for $\mathrm{NH}_{3}$ at different temperatures of deposition have been determined.

These values complete a lack of data at different temperatures from 13 up to $100 \mathrm{~K}$. Two different behaviours are obtained, in a first range of temperatures (from 13 to $60 \mathrm{~K}$ ), both $n$ and $\rho$ increase their values linearly with a rate of $0.00231 \mathrm{~K}^{-1}$ for $n$ and $0.00462 \mathrm{~g} \mathrm{~cm}^{-3} \mathrm{~K}^{-1}$ for $\rho$. Above $58 \mathrm{~K}$ a constant value is obtained for both $n$ and $\rho(n=$ $1.48 \pm 0.05$ and $\left.\rho=0.87 \pm 0.02 \mathrm{~g} \mathrm{~cm}^{-3}\right)$, in excellent agreement with the literature (see Figures 2 and 3). Specifically our value for the transition temperature from amorphous to crystalline phase 
obtained crossing the plateau with the straight line 391 fits is $58 \pm 5 \mathrm{~K}$.

The transition temperature deduced from our 393 procedure is almost the same than that obtained by 394 other authors (Dawes et al., 2007; Zheng \& Kaiser, 395 2007).

Despite this agreement, taking into account our 397 experimental error is not possible to discard a 398 metastable structure that has been a controversial 399 point during a long time. This metastable structure 400 was initially proposed by Staats \& Morgan (1959) 401 from the work of Reding \& Horning (1951), whose 402 results has been reproduced by many authors as re- ${ }_{403}$ vised Ferraro et al. (1980). This structure has also 404 been found by Moore and Hudson (1994) but they 405 called the range at which this features appear as 406 "transition phase". In order to elucidate the ap- 407 pearance or not of a metastable structure, more 408 recent works of Dawes et al. (2007) and Zheng \& 409 Kaiser (2007) have been designed with the aim of 410 clarifying whether this metastable phase of ammo- ${ }_{411}$ nia exists and, if is the case, the range of tempera- ${ }_{412}$ ture in which it is present.

Dawes et al. (2007) divide the dependence of ${ }_{414}$ the structure in two ranges: below and above 65 $\mathrm{K}$ where the transition occurs from amorphous to crystalline FCC. This study is based on the variation of the IR and Visible-UV spectrum of ammonia deposited at $25 \mathrm{~K}$ and comparing it with the spectra obtained at different temperatures during warming up. Additionally, they obtain spectra for $\mathrm{NH}_{3}$ deposited directly at higher temperatures and compare them with those deposited at low temper- ${ }_{419}$ atures and subsequently warmed up.

The study of Zheng \& Kaiser (2007) points out ${ }_{421}{ }^{220}$ the same behaviour but establishing the transition ${ }_{422}{ }_{421}$ point at slightly lower temperature: $58 \mathrm{~K}$. Despite ${ }^{423}$ these authors studied the sample only with the IR, ${ }^{424}$ their higher experimental resolution respect Dawes ${ }_{426}^{425}$ et al. (2007), consents them to show a fine struc- ${ }_{427}^{426}$ ture of the spectra appearing at $58 \mathrm{~K}$ that in- ${ }^{428}$ dicates structural changes associated to the crys- ${ }^{42}$ talline form.

Zheng \& Kaiser (2007) performed additional ex- ${ }^{432}$ periments using mixtures of ammonia with water ${ }^{433}$ ice, because water is the most important contami- ${ }^{434}$ nant in high vacuum systems and because its high ${ }^{435}$ reactivity with ammonia in the prechamber. They ${ }_{437}$ found that when ammonia is mixed with $1 \%$ of wa- ${ }^{438}$ ter, the transition temperature changes from 58 to $65 \mathrm{~K}$. These results show that the presence of water ${ }_{441}^{440}$ ice even as a contaminant, could vary the transition ${ }_{442}$ temperature from amorphous to crystalline.

Ice of carbon dioxide presents also a similar variation in temperature for $n$ and $\rho$, but presenting and increase with temperature higher than in the case of $\mathrm{NH}_{3}$ (Satorre et al., 2008). This lower variability in the case of ammonia could be associated to the H-bonds present in this molecule.

Our work is relevant because ammonia is abundant in many astrophysical scenarios. In those, some additional aspects must be taken into consideration: i) The buoyancy of mixtures when ammonia is present in a mixture liquid-solid context, will be greater than expected from their previous used value as its density could decrease down to $20 \%$ (as temperature decreases) from the usually assumed value of $1 \mathrm{~g} \mathrm{~cm}^{-3}$. ii) Irradiation dose (Moore et al., 2007) must be revised for experiments where $\mathrm{NH}_{3}$ is involved since penetration depth is affected by density of the film irradiated, therefore from our results ammonia could present values of penetration higher than expected. iii) Abundance calculated for the $\mathrm{NH}_{3}$ (Hudgins et al., 1993) in the scenarios where is present in solid state must also be revised considering our values for calculation purposes.

\section{Acknowledgments}

This work was supported by the Ministerio de Educación y Ciencia (Co-financed by FEDER funds) AYA 2009-12974.

\section{References}

Bauer J. M., Roush T. L., Geballe T. R., Meech K. J., Owen T. C., Vacca W. D., Rayner J. T., Jim K. T. C., 2002. The Near Infrared Spectrum of Miranda: Evidence of Crystalline Water Ice. Icarus 158, 178-190.

Bird M. K., Huchtmeier W. K., Gensheimer P., Wilson T. L., Janardhan P., Lemme, C. 1997. Radio detection of ammonia in comet Hale-Bopp. A\&A 325, L5-L8.

Born M., Wolf E., 1999. Principles of optics. Cambridge University Press.

Brown M. E., Calvin W. M. 2000. Evidence for Crystalline Water and Ammonia Ices on Pluto's Satellite Charon. Science 287, 107-109.

Cook J. C., Desch S. J., Roush T., Geballe T. R., Trujillo C. A. 2006. Near-Infrared Spectra of Charon: Support for Cryovolcanism on Kuiper Belt Objects? Lunar Planet. Sci. abstract 2107 .

Cook J. C.; Desch S. J.; Roush T. L.; Trujillo C. A.; Geballe T. R., 2007. Near-Infrared Spectroscopy of Charon: Possible Evidence for Cryovolcanism on Kuiper Belt Objects. ApJ 663, 1406-1419.

Dawes A., Mukerji R. J., Davis M. P., Holtom P. D., Webb S. M., Sivaraman B., Hoffmann S. V., Shaw D. A., Mason N. J., 2007. Morphological study into the temperature 
443 dependencee of solid ammonia under astrochemical condi- 508 444 tions using vacuum ultraviolet and Fourier-transform in- 509 445 frared spectroscopy. The journal of Chemical Physics 126, 510 $446 \quad 244711,1-12$

447 Dumas C.; Terrile R. J.; Brown R. H.; Schneider G.; Smith 51 448 B. A., 2001. Hubble Space Telescope NICMOS Spec- 513 449 troscopy of Charon's Leading and Trailing Hemispheres. 514 $450 \quad$ AJ $121,1163-1170$.

Emery J. P., Burr D. M., Cruikshank R. H., Brown R. H., 516 Dalton J.B., 2005. Near-infrared (0.8-4.0 m) spectroscopy 517 of Mimas, Enceladus, Tethys, and Rhea. Astron. Astro- 518 phys. 435, 353-362.

Ferraro J. R., Sill G., Fink U., 1980. Infrared Intensity Mea- 520 surements of Cryodeposited Thin Films of $\mathrm{NH}_{3}, \mathrm{NH}_{4} \mathrm{HS}, \quad{ }_{21}$ $\mathrm{H}_{2} \mathrm{~S}$, and Assignments of Absorption Bands, App. Spec- 522 troscopy 34, 525-533.

d'Hendecourt L. B., Allamandola L. J., 1986. Time de- ${ }^{524}$ pendent chemistry in dense molecular clouds. III-infrared 525 band cross sections of molecules in the solid state at 1052 K. A\&ASS 64, 453-467.

Heavens O. S., 1991. Optical properties of thin solid films. ${ }_{528}$ Dover publications, Inc.

Hudgins D. M., Sandford S. A., Allamandola L. J., Tielens 530 A. G. G. M., 1993. Mid and far-infrared spectroscopy of 531 ices: optical constants and integrated absorbances. ApJSS 532 $86,713-870$.

Jewitt D. C., Luu J. 2004. Crystalline water ice on the 534 Kuiper belt object (50000) Quaoar. Nature 432, 721-733. 535

Kargel J. S., 1991. Brine volcanism and the interior struc- 536 tures of asteroids and icy satellites. Icarus 94, 368-390. 537

Kargel, J. S., 1992. Ammonia-water volcanism on icy satellites: Phase relations at 1 atmosphere. Icarus 100, 556574 .

Kawakita H., Watanabe J. 2002. Revised Fluorescence Efficiencies of Cometary $\mathrm{NH}_{2}$ : Ammonia Abundance in Comets. ApJ 572, L177-L180.

Leliwa-Kopystynski J., Maruyama M., Nakajima T. 2002. The water-ammonia phase diagram up to $300 \mathrm{MPa}$ : Application to icy satellites. Icarus $159,518-528$.

Lewis J. S., 1972. Low Temperature Condensation from the Solar Nebula. Icarus, 16, 241-252.

Lodders, K., 2003. Solar system abundances and condensation temperatures of the elements. ApJ 591, 1220-1247.

Manzhelii V. G., Tolkachev A. M., 1964. Densities of ammonia and methane in the solid state. Soviet physics-Solid state 5, 12, 2506-2510.

Marion G. M., Kargel J. S., Catling D. C., Lunine J. L., 2012. Modeling ammonia-ammonium aqueous chemistries in the Solar System's icy bodies. Icarus 220, 932-946.

Moore M. H., Hudson R. L., 1994. Far-infrared spectra of cosmic-type pure and mixed ices. A\&ASS 103, 45-56.

Moore, M. H.; Ferrante, R. F.; Hudson, R. L.; Stone, J. N., 2007. Ammonia water ice laboratory studies relevant to outer Solar System surfaces. Icarus 190, 260-273.

Olovsson I., Templeton D., 1959. X-ray study of solid ammonia. Acta Cryst. 12, 832-836.

Pipes J. G., Roux J. A., Smith A. M., 1978. Infrared transmission of contamined cryocooled optical windows. AIAA Journal 16, 9, 984-990.

Pizzarello S., Williams L. B., 2012. Ammonia in the Early Solar System: An Account from Carbonaceous Meteorites. ApJ 749, 161-166.

Reding F. P., Horning D. F., 1951. The Vibrational Spectra of Molecules and Complex lons in Crystals. V. Ammonia and Deutero-Ammonia, J. Chem. Phys. 19, 594-601.
Romanescu C., Marxchall J., Kim D., Khatiwada A., Kalogerakis K. S., 2010. Refractive index measurements of ammonia and hydrocarbon ices at $632.8 \mathrm{~nm}$. Icarus 205, 695-701.

Satorre M. Á., Domingo M., Millán C., Luna R., Vilaplana R., Santonja C., 2008. Density of $\mathrm{CH}_{4}, \mathrm{~N}_{2}$ and $\mathrm{CO}_{2}$ ices at different temperatures of deposition. P\&SS 56, 17481752.

Staats P. A., Morgan H. G., 1959. Infrared Spectra of Solid Ammonia, J. Chem. Phys. 31, 553-554.

Tempelmeyer K. E. , Mills Jr. D. W., 1968. Refractive index of carbon dioxide cryodeposits. J. Appl. Phys. 39, 29682969.

Verbiscer A. J., Peterson D. E., Skrutskie M. F., Cushing M., Helfenstein P., Nelson M. J., Smith J. D., Wilson J. C. 2006. Near-infrared spectra of the leading and trailing hemispheres of Enceladus. Icarus 1852, 211-223

Westley M. S., Baratta G. A., Baragiola R. A., 1998. Density and index of refraction of water ice films vapor deposited at low temperatures. Journal of Chemical Physics 108, 8, 3321-3325.

Wood B. E., Roux J. A., 1982. Infrared optical properties of thin $\mathrm{H}_{2} \mathrm{O}, \mathrm{NH}_{3}$, and $\mathrm{CO}_{2}$ cryiofilms. J. Opt. Soc. Am. 72, 6, 720-728.

Zheng W., Kaiser R., 2007. An infrared spectroscopy study of the phase transition in solid ammonia. Chemical Physics Letters 440, 229-234.

Ziegler J. P., Biersack J.P., Litmark, U., 1985. The stopping and range of ions in solids. Pergamon, New York, See also http://www.srim.org. 